\title{
MINORÍAS ÉTNICAS EN EL REINO UNIDO. EJEMPLO DE SHEFFIELD
}

\author{
Teresa RICA PÉREZ
}

Becaria Erasmus del Departamento de Geografía y Ordenación del Territorio Universidad de Zaragoza

\begin{abstract}
Resumen: El estudio de las minorías étnicas establecidas en las grandes ciudades europeas preocupa a los geógrafos, economistas, sociólogos y políticos por las implicaciones de todo orden que las minorías étnicas tienen en la vida colectiva. El estudio de estas minorías en la ciudad de Sheffield (Reino Unido), pone de manifiesto la diversidad étnica, demográfica, socio-económica y cultural e incluso espacial de su población, todo lo cual constituye un componente muy representativo de la nueva sociedad que se está configurando en las áreas urbanas europeas como resultado de la inmigración.
\end{abstract}

Palabras clave: Minorías étnicas, migraciones, segregación/concentración espacial.

\begin{abstract}
The study of the ethnic minority communities in the city of Sheffield shows the ethnic, demographic, socio-economic and culturel diversity, what is more the spatial diversity of her population. These constitute a very representative component of the new society which is forming in the european urbane metropolitan areas/regions as a result of the immigration.
\end{abstract}

Key Words: Ethnic minority communities, the migrations, spatial segregation/concentration.

\section{INTRODUCCION}

La movilidad espacial de la población constituye hoy un aspecto de importancia fundamental en la Geografía de la Población como determinante de procesos de crecimiento o declive en ciudades, regiones o incluso países, bien a través de la movilidad residencial, o indirectamente por sus efectos en el crecimiento natural, y por tanto en la estructura y composición de la población, así como por su incidencia en diversos aspectos de la vida cotidiana: mercado de viviendas, servicios, economía, etc. (CHAMPION \& FIELDING, 1992). 
El carácter heterogéneo de la población de las ciudades europeas desde el punto de vista de su composición es el resultado de diversas fases inmigratorias, cuyas causas son demográficas y socioeconómicas, y dan origen a diferentes grados de integración social y espacial. Como aportación al estudio de las minorías étnicas en Europa presentamos el ejemplo de Sheffield (R.U.)

Los flujos migratorios han configurado en algunos países, como sucede en el Reino Unido, una población heterogénea cuyas características étnicas se recogen en el censo. A una mayoría de población, censada como White, se suman, en proporciones variables, diversos grupos étnicos, ampliamente representados en las áreas urbanas. La declaración de pertenencia a un grupo étnico concreto constituye, pues un elemento más de caracterización de la población en el Censo de 1991.

Ejemplo de esa heterogeneidad que se refleja en el Censo de población británico de 1991, es la ciudad de Sheffield donde junto a un grupo mayoritario que representa el $94^{1} 96 \%$ de la población total, denominado White, hay una serie de minorías configuradas por etnias originarias de países africanos, asiáticos y americanos ${ }^{1}$.

Sin embargo, la denominación étnica no coincide siempre con el lugar de nacimiento, pues en todos los grupos censados, se encuentran personas nacidas dentro y fuera del Reino Unido, cuyo origen étnico evidencia una importante movilidad internacional.

Es sabido que la movilidad de la población puede clasificarse atendiendo a su duración, razones que la provocan, personas y áreas afectadas, etc. Estas categorías no son excluyentes, pues hay migraciones efímeras y definitivas, voluntarias y forzosas, rápidas y lentas, en masa o individuales y espontáneas u organizadas, según sean las circunstancias personales de los desplazados. No obstante, hay dos tipos principales de motivaciones que impulsan los flujos de población: económicas y socio-políticas. Las primeras ligadas, sobretodo, a la búsqueda de mejores oportunidades laborales, y las segundas, a conflictos que pueden ir vinculados a enfrentamientos bélicos.

\footnotetext{
1 Población incluída en los grupos étnicos considerados en el Censo de población del Reino Unido, en 1991:

- White: White (griegos, turcos, otros europeos) y White mixtos.

- Black-Caribbean: del Caribe, Guyana e Indias Occidentales.

- Black-African: del continente africano (excepto del Norte de Africa).

- Black-Other: Black ( ingleses y otros), Black/White, Black "mixtos".

- Indian: Hindúes.

- Pakistani: Pakistaníes.

- Bangladeshi: Bangladesis.

- Chinese: Chinos.

- Other Asian.

- Otros: Norte de Africa, Arabia o Iran, White/Black/White de Asia.
} 
En ocasiones las relaciones históricas y políticas tradicionalmente existentes entre el lugar de origen -colonias- y el de destino de los migrantes -metrópolis-, han sido un factor importante, aunque no exclusivo, de la movilidad internacional. En el caso del Reino Unido, los lazos históricos desempeñan un papel de primer orden puesto que ha sido una nación colonizadora, que ha atraído hacia sí inmigrantes desde las colonias con flujos variables en su carácter e intensidad a lo largo del tiempo. En los últimos cincuenta años, cabría identificar los máximos flujos migratorios hacia el Reino Unido en tres 'olas de movimiento' en las que se diferencian varios procesos (Fig. 1).

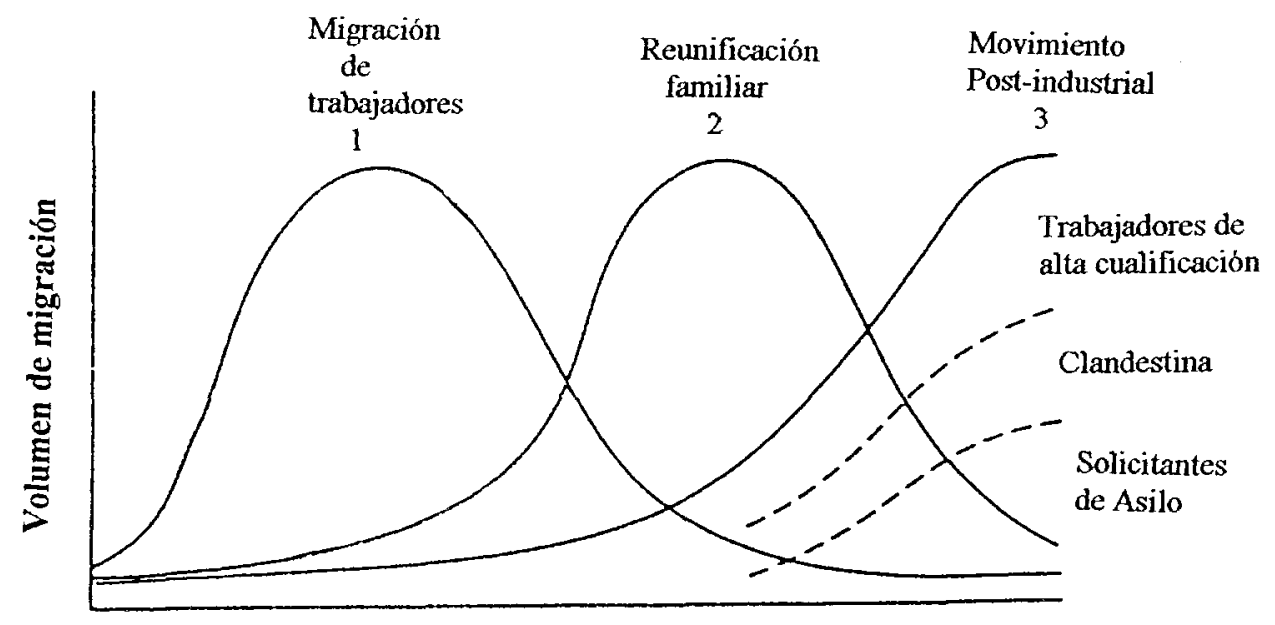

Tiempo

Figura 1.- Oleadas de inmigración internacional en ciudades europeas. Fuente: WHITE (1993).

Después de la II Guerra Mundial, el Reino Unido ha soportado dos grandes olas de inmigraciones internacionales: la "Migración de trabajadores" (Labour migration); y la "De reunificación familiar" (The family reunification). En la actualidad se está produciendo la llamada "Ola de movimiento post-industrial "(The wave of the postindustrial movement).

La cresta de la primera ola se alcanzó entre 1950 y 1970, y se caracterizaba por sus conexiones coloniales y la gran diversidad de procedencia de los inmigrantes. Se trataba fundamentalmente de trabajadores que dejaron a sus familias en el país de origen. La segunda ola comenzó hacia 1970, produciéndose en muchos casos en un corto espacio de tiempo. Y la tercera, conocida como "De movimiento post-industrial", que se está produciendo actualmente, presenta, a su vez, tres corrientes principales: la de 
trabajadores de alta cualificación, las migraciones clandestinas, y las migraciones de refugiados procedentes del Este de Europa y de países subdesarrollados.

Las principales comunidades étnicas establecidas en el Reino Unido no llegaron simultáneamente. Los inmigrantes de origen afro-caribeño llegaron masivamente entre 1955 y 1960 para suplir la falta de mano de obra en la industria del acero y en otros sectores; los procedentes de Pakistán habían comenzado a llegar antes de la Segunda Guerra Mundial, aunque el máximo de entradas se produjo de 1950 a 1960.

En los diferentes países de la Unión Europea, el rasgo común de las migraciones es que los inmigrados se asientan principalmente en las grandes ciudades y en las regiones industriales. Y lo mismo sucede con la inmigración clandestina, que tiene en las ciudades más oportunidades de encontrar un trabajo y de confundirse entre 1 a población total.

La presencia de inmigrantes origina en las áreas receptoras tres respuestas diferentes: a) una aparente asimilación y disolución de la propia identidad; b) la formación de grupos étnicos que se constituyen como grupo diferente de la población mayoritaria; y c) el desarrollo de una sociedad pluralista.

A medida que ha ido aumentando el número de inmigrantes se ha podido comprobar que no hay coincidencia entre los deseos de los mismos y las políticas de inmigración que generalmente son contradictorias, como se ha puesto de manifiesto en los acuerdos tomados en la Unión Europea:"El establecimiento de un mercado único interno conlleva factores financieros y comerciales a expensas de los derechos humanos" (BAIMBRIDGE, 1994).

\section{POBLACION TOTAL Y GRUPOS ETNICOS}

El Censo de 1991 registra en la ciudad de Sheffield una población inscrita total de 501.202 habitantes, en los que se diferencian varios grupos étnicos. Presentar una clasificación étnico-racial no es habitual en censos realizados en otros países como España, aunque sí en el Reino Unido.

Fn la ciudad de Sheffield, el grupo étnico denominado White con 475.977 habitantes es claramente mayoritario mientras el 5'04\% restante de la población, lo constituyen otros grupos étnicos: Black Caribbean, Black African, Other Black, Indian, Pakistani, Bangladeshi, Chinese, Other Asian, Other Ethnic Groups y Born in Ireland, siendo conocidos, debido a su menor presencia numérica, como minorías étnicas (Ethnic minority communities) (Fig. 2). 


\begin{tabular}{|l|c|}
\hline MINORIAS ETNICAS & PORCENTAJE(\%) \\
\hline BLACK CARIBBEAN & 17.46 \\
\hline BLACK AFRICAN & 3.8 \\
\hline OTHER BLACK & 6.53 \\
\hline INDIAN & 4.93 \\
\hline PAKISTANI & 30.98 \\
\hline BANGLADESHI & 3.77 \\
\hline CHINESE & 4.64 \\
\hline OTHER ASIAN & 3.3 \\
\hline OTHER ETHNIC GROUPS & 12.55 \\
\hline BORN IN IRELAND & 12.04 \\
\hline
\end{tabular}

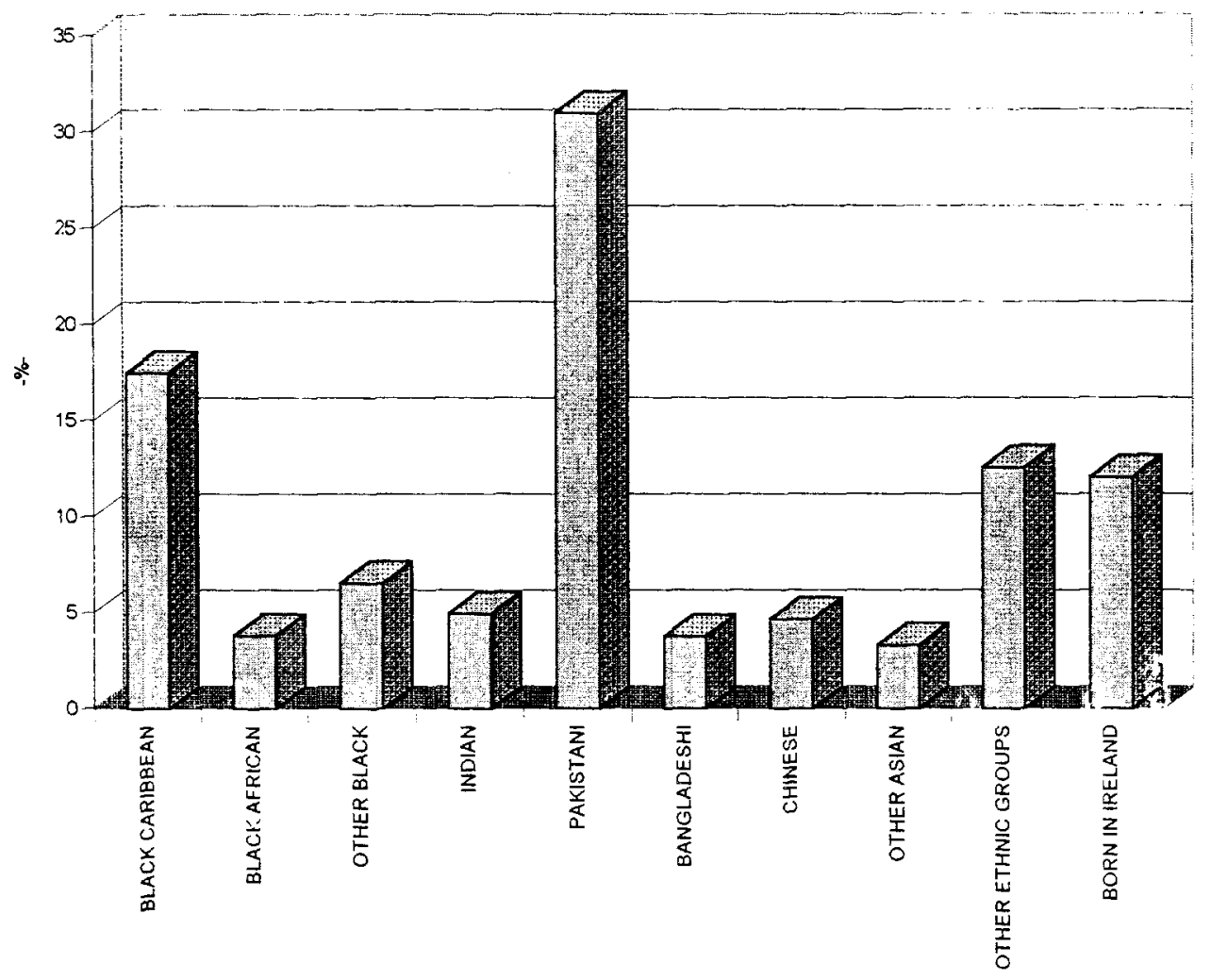

Figura 2.- Grupos y minorías étnicas en Sheffield .

Fuente: VV.AA. (1991): Sheffield 1991. Census. Elaboración propia.

Los porcentajes se refieren al total de población de las minorías étnicas, no considerando al grupo white. 
La superioridad en número del primer grupo no debe restar importancia a la elevada diversidad étnica que caracteriza a la población de la ciudad, y que influye de modo evidente en las tendencias actuales de la evolución demográfica que son muy contrastadas, con tendencia al estancamiento y envejecimiento en el grupo predominante, y conservando un carácter joven los grupos minoritarios.

Entre los grupos minoritarios destacan por su número los llamados Pakistani y los Black Caribbean que son el $30^{\prime} 98 \%$ y el $17^{\prime} 46 \%$ respectivamente del total de población de las minorías étnicas, mientras que el resto de los grupos varían desde el mínimo del 3'30\% de Other Asian hasta un 12'55\% de Other Ethnic Groups.

Atendiendo al lugar de nacimiento, sin tener en cuenta la clasificación étnica establecida en el censo de la población de Sheffield, se aprecia que la proporción de nacidos en el Reino Unido, incluyendo los nacidos en la República de Irlanda y en otras islas británicas, es muy elevada alcanzando al 96'30\%. Eso nos permite conocer el número real de entradas que, según el último censo, significan solamente el $3^{\prime} 70 \%$ de 1 a población total, o lo que es lo mismo 18.553 habitantes.

De éstos, los nacidos en territorios de la Commonwelth, un total de 11.465 personas, son los más numerosos seguidos, a cierta distancia, por los nacidos en Europa, 3.141, en países asiáticos, 2.086, y africanos, 999. De los procedentes de la Commonwelth ocupan un lugar importante los de Pakistán, 4.296 personas, seguidos de los de Jamaica, 2.007, India, 1.070, y Bangladesh, 664, y entre los de otras procedencias destacan los procedentes de Alemania, 111; República de Sudáfrica, 285; EE.UU, 385, y República Popular China, 339 personas.

En ambos casos, bien según la clasificación étnico-racial del censo o según el lugar de nacimiento, sobresale como grupo étnico relativamente homogéneo, el White, con personas en su mayoría nacidas en el Reino Unido, frente a la gran diversidad de las llamadas minorías étnicas entre las que destacan las Comunidades Black Caribbean y Pakistani.

En síntesis, las categorías más representadas en los grupos étnicos son:

-Los nacidos en el Reino Unido en los denominados White ya que representan el 98 ' $2 \%$ de toda la población de este grupo étnico y Other Black, ya que el $94 ' 21 \%$ de la población de este último grupo étnico ha nacido en U.K.

-Los nacidos fuera del Reino Unido entre los grupos Other Asian, Chinese, Black African, Bangladeshi, e Indian, representan el 8051\%, 75'29\%, 65\% $89 \%$, $6152 \%$ y $6067 \%$ respectivamente del total de población de cada grupo. 
-Con proporción equivalente de nacidos fuera y en el Reino Unido, Black Caribbean (45'34\% de la población de este grupo étnico ha nacido fuera y $5466 \%$ ha nacido en U.K) y Pakistani (48'2\% de la población de este grupo ha nacido fuera y 51 ' $8 \%$ ha nacido en el Reino Unido).

Las procedencias son pues equivalentes en los dos grupos étnicos minoritarios más representados, Black Caribbean y Pakistani, mientras otros grupos más minoritarios apoyan su población, unas veces, en la alta proporción de llegadas como sucede con la Comunidad China , y otras en los nacidos en el Reino Unido como el grupo étnico Other Black. Todo lo cual configura un mosaico heterogéneo con implicaciones de todo orden.

\section{CARACTERISTICAS DEMOGRAFICAS DE LOS GRUPOS ETNICOS}

Un rasgo fundamental que diferencia el grupo White y el de las Comunidades consideradas minoritarias es su perfil demográfico. La estructura demográfica podría calificarse de estable en el primero, siendo por el contrario irregular en los otros grupos, lo cual tiene mucho que ver con la inmigración. Pero su perfil estructural no es uniforme ya que cada 'Comunidad étnica' se ha constituído como resultado de corrientes de entrada ocurridas en momentos diferentes y generadas en un contexto político, económico y social también diferente, y tienden a conservar su perfil estructural originario, al menos durante un par de generaciones (Fig. 3).

EI mayor equilibrio numérico entre los diferentes grupos de edades diferencia el perfil estructural del grupo White, afectado, a su vez, por una base regresiva y una sobrerrepresentación de los grupos de 20 a 24 y de 25 a 29 años, como resultado del mayor número de nacimientos ocurridos en la década de los 60. Todas las Comunidades consideradas minorías étnicas se caracterizan por pirámides muy irregulares, destacando en algunos casos los máximos valores en los grupos de edades más jóvenes, lo que puede considerarse como una prueba de perfil progresivo, o lo que es lo mismo, el hecho de que la población joven está presente en mayor proporción que la población adulta y de mediana edad. Esto sucede en las Comunidades Bangladeshi, Black Other, Other Ethnic Groups y Pakistani, como resultado de la conjunción de varios procesos:

a.- De un lado, por la reagrupación familiar de trabajadores anteriormente inmigrados lo que dio origen a una segunda generación de inmigrantes e incluso a una tercera, como es el caso de la Comunidad Pakistaní, 
b.-De otro, como resultado de movimientos de familias de exiliados que han mantenido una alta natalidad -como es el caso de la Comunidad Somali-.
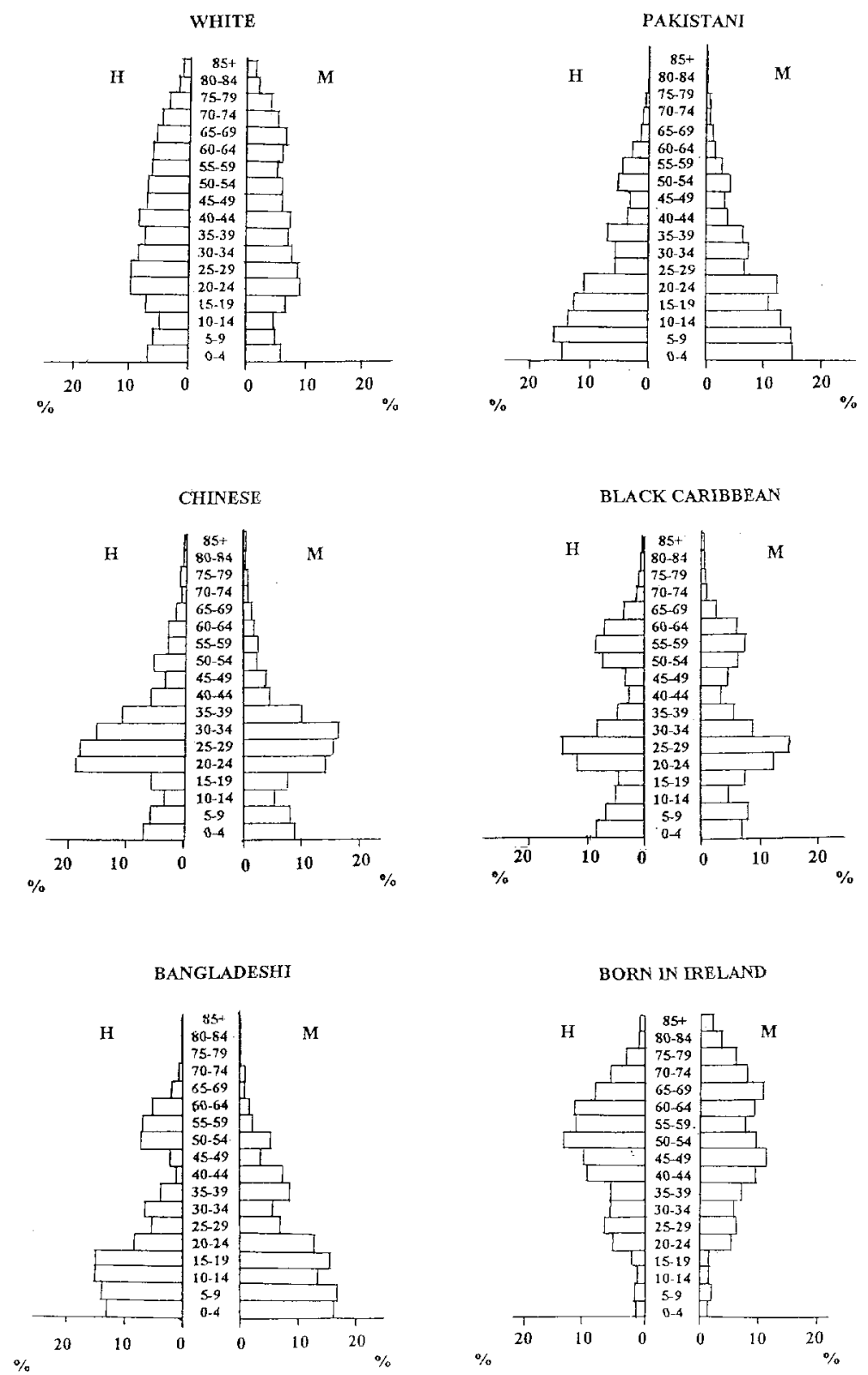

Figura 3.- Estructura demográfica en grupos étnicos de la ciudad de Sheffield Fuente: Censo, 1991. 
Sin embargo, en otras Comunidades, la Comunidad China, Hindú, Black Caribbean, Black African y Other Asian Groups, los grupos de edades más jóvenes no son los más destacados, como resultado de fenómenos particulares en su evolución. Así los máximos de población de 20-24 años y 30-34 en la Comunidad China, unido al elevado número de los no nacidos en el Reino Unido, se explican por la entrada de estudiantes chinos que acuden al Reino Unido a realizar sus estudios universitarios. En el caso de la Comunidad Black-Caribbean, la situación es otra, los máximos se dan en los grupos de 50-59 y de 25-29 años. El grupo de mas edad se identifica con la oleada inicial de los años 50 y 60 atraída por la escasez de mano de obra en las industrias, y el más joven está compuesto por la segunda generación o nacidos ya en el país de destino.

De ese modo, aunque se puede hablar de un primer rasgo que diferencia a 1 a Comunidad White, mas estable y con mayor tendencia al envejecimiento que el resto de la población, no se puede agrupar al resto de los grupos étnicos por características homogéneas.

Las relaciones de dependencia son muy desiguales según predomine una población joven, adulta o envejecida. Entre las Comunidades que presentan unas relaciones de dependencia mas elevada destacan los Other Black, Pakistani y Bangladeshi, debido a lo elevada que es su población joven, la población de 0 a 15 años representa en cada una de ellas respecto a sus totales de población, el 59'52\%, 43'76\% y $43^{\prime} 98 \%$ respectivamente, mientras que en otras Comunidades esta relación es baja como sucede en la Comunidad China y el grupo constituído por Otros Asiáticos, con el 75'71\% y $7278 \%$ de adultos (16 a 65 años) respectivamente respecto a su población total. La Comunidad White mantiene una posición intermedia con $6076 \%$ de población de 16 a 65 años y $1740 \%$ de población joven menor de 15 años. Eso significa una menor disposición teórica de población activa en la Comunidad White en un futuro próximo y un aumento en la dependencia por envejecimiento de la población. Si a ello añadimos que la Comunidad White es el 94'96\% de la población total de la ciudad, habrá que plantear necesariamente una mejora o aumento de servicios para esa creciente población de mas edad sin por ello descuidar los necesarios para la población joven con la que aún cuentan en mayor proporción algunas de las Comunidades minoritarias.

Si hay diferencias numéricas importantes entre los grupos de edad de las minorías étnicas, la sex-ratio es equivalente. La relación de masculinidad (sex-ratio masculina) observada oscila entre $47^{\prime} 28 \%$ para Other Asian y $56^{\prime} 90 \%$ para Other Ethnic Groups.. No hay por tanto sobrerrepresentación significativa de la población de uno u otro sexo en dichas Comunidades. y como la Comunidad White es la más numerosa su ratio de masculinidad pesa mucho en la de la población total de Sheffield que es de $4829 \%$, mientras el número de varones es superior entre Black Caribbean 
(51'88\%), Black African (55'31\%), e Indian (51'57\%), debido a las características de la inmigración.

En resumen, se puede hablar de diferencias demográficas contrastadas entre la poblaciónWhite y los diversos grupos étnicos minoritarios producto de las principales corrientes inmigratorias a las que se suman las nuevas generaciones ya nacidas en el país.

\section{CARACTERISTICAS SOCIALES, ECONOMICAS Y CULTURALES}

Las denominadas minorías étnicas presentan algunas particularidades desde el punto de vista de sus características socioeconómicas y socioculturales:

Se reconocen rasgos diferenciales por sus características socioeconómicas entre los grupos étnicos así como la evolución de los mismos desde la primera a la segunda generación (los nacidos en el Reino Unido más los que llegaron al país con menos de cinco años).

En lo que se refiere al estado civil es frecuente encontrar en algunos grupos, como sucede con las Comunidades White o Black-Caribbean, situaciones de cohabitación y separaciones matrimoniales, que son en cambio inusuales en algunos grupos de origen asiático como el Pakistani y Bangladeshi, especialmente por lo que se refiere a las mujeres. En el fondo se trata de un comportamiento de raíz cultural, que ha sido constante tanto en la primera como en la segunda generación. Fn otras categorías de estado civil como las de casados y solteros, tiende a descender el número de casados y a aumentar el de solteros tanto en los asiáticos, en particular las segundas generaciones (Pakistanies, Hindues e hijos de Bangladeshis), como en la comunidad White.

Por lo que se refiere a la unidad familiar también hay diferencias entre grupos étnicos puesto que matrimonio con hijos son más frecuentes entre Pakistaníes, Bangladeshis e Hindúes que en los White, Caribbean y Black-African, ya que mantienen los comportamientos reproductivos de sus lugares de origen.

Finalmente, atendiendo a la frecuencia de uniones interétnicas, estas se llevan a cabo en mayor medida entre los Caribbean yWhite, que entre éstos e Hindúes, Pakistanies o Bangladeshis. Hay por tanto menor propensión a uniones interétnicas en algunas Comunidades, así las mujeres de origen Hindú, Pakistaní y Bangladeshi no se unen habitualmente con miembros de la ComunidadWhite, probablemente por "reparos" culturales. 
-SHEFFIELD 1991-

\begin{tabular}{|l|c|c|l|c|c|}
\hline VARONES & ACTIVOS(\%) & INACTIVOS(\%) & MUJERES & ACTIVOS(\%) & INACTIVOS(\%) \\
\hline White & 70.18 & 29.82 & White & 47.97 & 52.03 \\
\hline Black Caribbean & 76.54 & 23.46 & Black Caribbean & 64.32 & 35.68 \\
\hline Black African & 59.12 & 40.88 & Black African & 43.09 & 56.91 \\
\hline Black Other & 80.39 & 19.61 & Black Other & 61.82 & 38.18 \\
\hline Indian & 70.3 & 29.7 & Indian & 56.64 & 43.36 \\
\hline Pakistani & 71.18 & 28.82 & Pakistani & 25 & 75 \\
\hline Bangladeshi & 66.05 & 33.95 & Bangladeshi & 25.44 & 74.56 \\
\hline Chinese & 51.42 & 48.58 & Chinese & 43.84 & 56.16 \\
\hline Other Asian & 42.1 & 57.9 & Other Asian & 31.45 & 68.55 \\
\hline Other Groups & 62.99 & 37.01 & Other Groups & 44.72 & 55.28 \\
\hline Born in Ireland & 64.81 & 35.19 & Born in Ireland & 46.32 & 53.68 \\
\hline Total & 70.1 & 29.9 & Total & 47.82 & 52.18 \\
\hline
\end{tabular}

-POBLACION MASCULINA ACTIVA E INACTIVA-

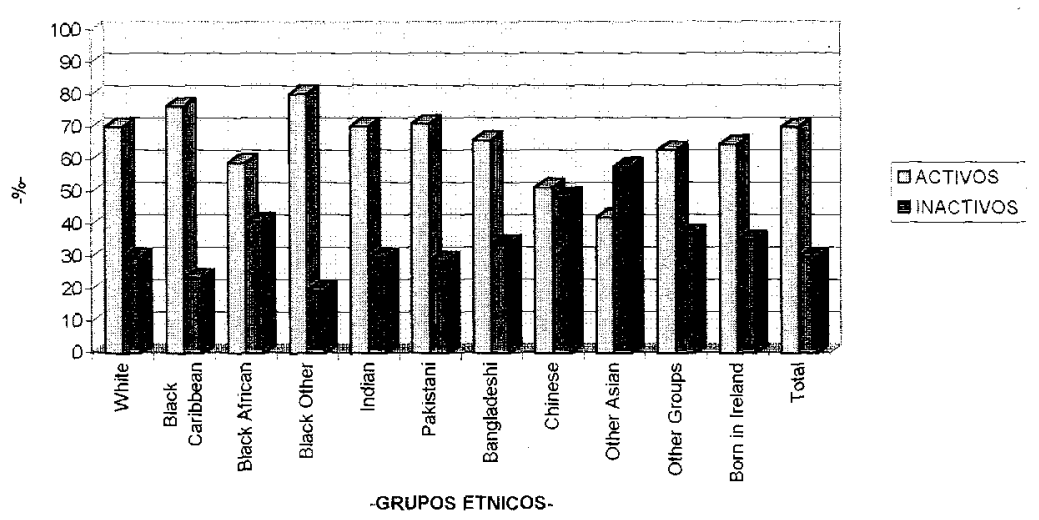

-POBLACION FEMENINA ACTIVA E INACTIVA-

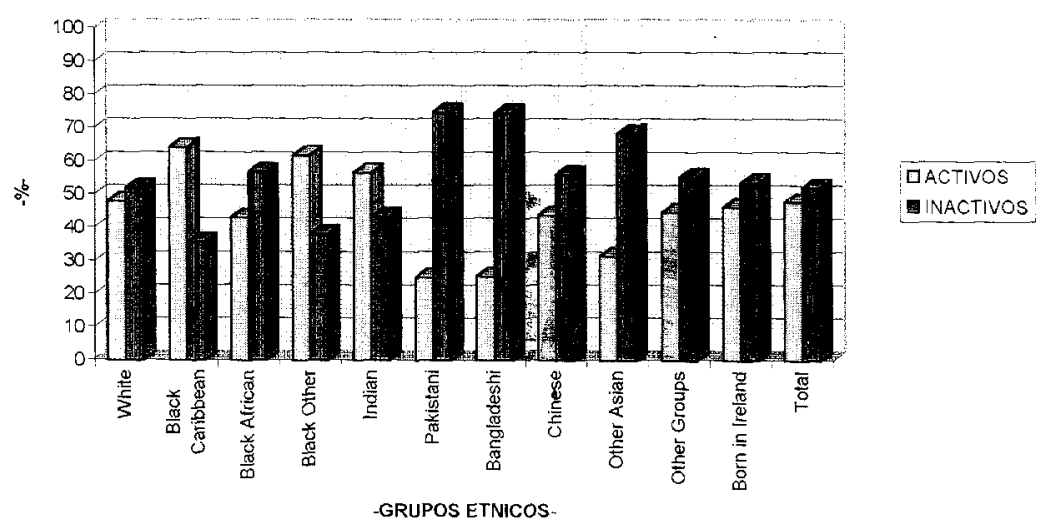

Figura 4.- Población activa e inactiva por sexos en los grupos étnicos. Fuente: Sheffield Census-1991. Elaboración propia. 
Desde el punto de vista de sus características económicas, el estudio de la población activa e inactiva en los grupos étnicos minoritarios pone de manifiesto que las mayores proporciones de población activa masculina se encuentran en la Comunidad Black Caribbean (76'54\%), Black Other (80'39\%), Pakistani( $71^{\prime} 18 \%$ ) y White $(7018 \%)$, a cierta distancia de los valores más bajos en las comunidades Chinese $\left(51^{\prime} 42 \%\right)$ y Other Asian $\left(42^{\prime} 10 \%\right)$. Sin embargo, si atendemos a la diferenciación por sexos no siempre se corresponden estos valores ya que aunque la Comunidad Pakistani tiene una elevada población activa masculina, la femenina es escasa. Siendo los menores valores de población activa femenina los de la Comunidad Pakistani (25\%) y Bangladeshi $\left(25^{\prime} 44 \%\right.$ ) y a cierta distancia Indian (56'64\%) y Black Other $\left(61^{\prime} 82 \%\right)$ (Fig. 4).

En cuanto a su ocupación los hombres que trabajan a tiempo completo predominan en la Comunidad White, Born in Ireland, Other Asian y Chinese, pero son en cambio las mujeres en la Comunidad Black Caribbean o en la Black Other. Del mismo modo los hombres predominan como trabajadores a tiempo parcial, en la Comunidad Black African y Bangladeshi, pero las mujeres en la Comunidad White. Afectando del mismo modo la categoría de desempleados tanto a hombres como a mujeres en los grupos Black African, Pakistani y Bangladeshi fundamentalmente.

La destacada proporción de población jubilada en el total de población inactiva de la Comunidad White y de los Born in Ireland, y un elevado índice de estudiantes, tanto varones como mujeres, de las Comunidades China, India, Black African y Other Asian., constituyen el grueso de la población inactiva.

La diversidad cultural es enorme si atendemos a algunas de sus manifestaciones mas importantes como es la lengua utilizada. Entre las minorías étnicas de Sheffield se han contabilizado hasta 47 lenguas diferentes. La lengua inglesa es la oficialmente reconocida y la hablada normalmente por la población, lo que no excluye el uso de otras lenguas. De la gran variedad de lenguas habladas, sobresalen la Punjabi-Lrdu, Bengali, Somali, Arabe , Creole-Patois y Cantonese, que en 1992 eran habladas por 1.866 alumnos bilingües en Educación Secundaria en la ciudad de Sheffield ${ }^{2}$. A pesar de que las consideradas minorías étnicas representan una pequeña parte de la población total, un número importante de organizaciones, se contabilizaron 26, están vinculadas a los diversos grupos étnicos y constituyen un vehículo de apoyo a la lengua y cultura propia

\footnotetext{
2 Lenguas de uso frecuente en Sheffield, aparte de la oficial, y procedencia de la población que las utiliza - Punjabi-Urdu: India, Pakistán.

- Bengali: Bangladesh y Oeste de Bengala-India.

- Somalí: Somalia.

- Arabe: Yemen, Iraq, Irán, Palestina y algunos somalíes.

- Creole-Patois: Islas del Caribe, Jamaica.

- Cantonese: China.
} 
de cada grupo, llegando a contar con mas de 1.000 alumnos, jóvenes fundamentalmente, durante el curso 1994-95.

\section{LOCALIZACION ESPACIAL DE LOS GRUPOS ETNICOS EN LA CIUDAD}

Desde el punto de vista de la Geografía es de gran interés conocer además de las características demográficas y socioeconómicas propias de estas poblaciones, su distribución y efectos en el espacio.

En el caso de Sheffield las diversas minorías étnicas están presentes en mayor o menor medida en cada una de las áreas administrativas en que está dividida la ciudad, aunque cada Comunidad tiende a concentrarse en unas áreas residenciales más específicas. La Comunidad White se localiza preferentemente en toda la periferia urbana (SE, SW, NE y NW) y disminuye su presencia hacia el centro de la ciudad. E1 grupo Black-Caribbean se localiza fundamentalmente en las áreas del Centro-Este urbano, sin que se produzca necesariamente segregación étnico-espacial ya que hay Black-Caribbean viviendo en las áreas donde predomina la población de la Comunidad White y viceversa. Lo mismo sucede en el resto de los grupos étnicos que aunque están distribuídos por toda la ciudad dominan en algunas áreas (Fig. 5).

La localización de Black-African coincide con la del grupo Black-Caribbean, a 1 igual que sucede con el grupo Other Black, de manera que se concentra el grupo Black en barrios como el de Castle o Burngreave en el sector centro oriental de la ciudad.

Los grupos étnicos de origen asiático, Bangladeshi y Pakistani, coinciden en algunas áreas del Este, Darnall, Burngreave, Brithside o Firth Park -principalmente-, pero no siempre es así pues la Comunidad Bangladesh tiene además otra área de importante localización, el barrio de Hallam, al SW. Igualmente, las Comunidades Indian y Chinese comparten con la Bangladeshi el SW de la ciudad en la proximidad de la Comunidad White como sucede con el barrio de Ecclessall para White e Indian o con el área de Mosborough para White y Chinese, en el SE de Sheffield. Como resultado se están configurando áreas urbanas caracterizadas por una cultura mixta con predominio de uno u otro grupo étnico-cultural.

Los Other Ethnic Groups, se localizan entre Comunidades asiáticas y black, configurándose unos espacios de proximidad y contacto entre grupos de diferentes continentes - Asia, Africa y América-.

Finalmente hay diferentes comportamientos de localización dentro de una misma Comunidad ya que si se considera a los de origen irlandés como lo hace el Censo 
de 1991, se comprueba que no coincide exactamente con la localización de la Comunidad White sino con la Black y Asian en las áreas de Burngreave, Sharrow, etc.
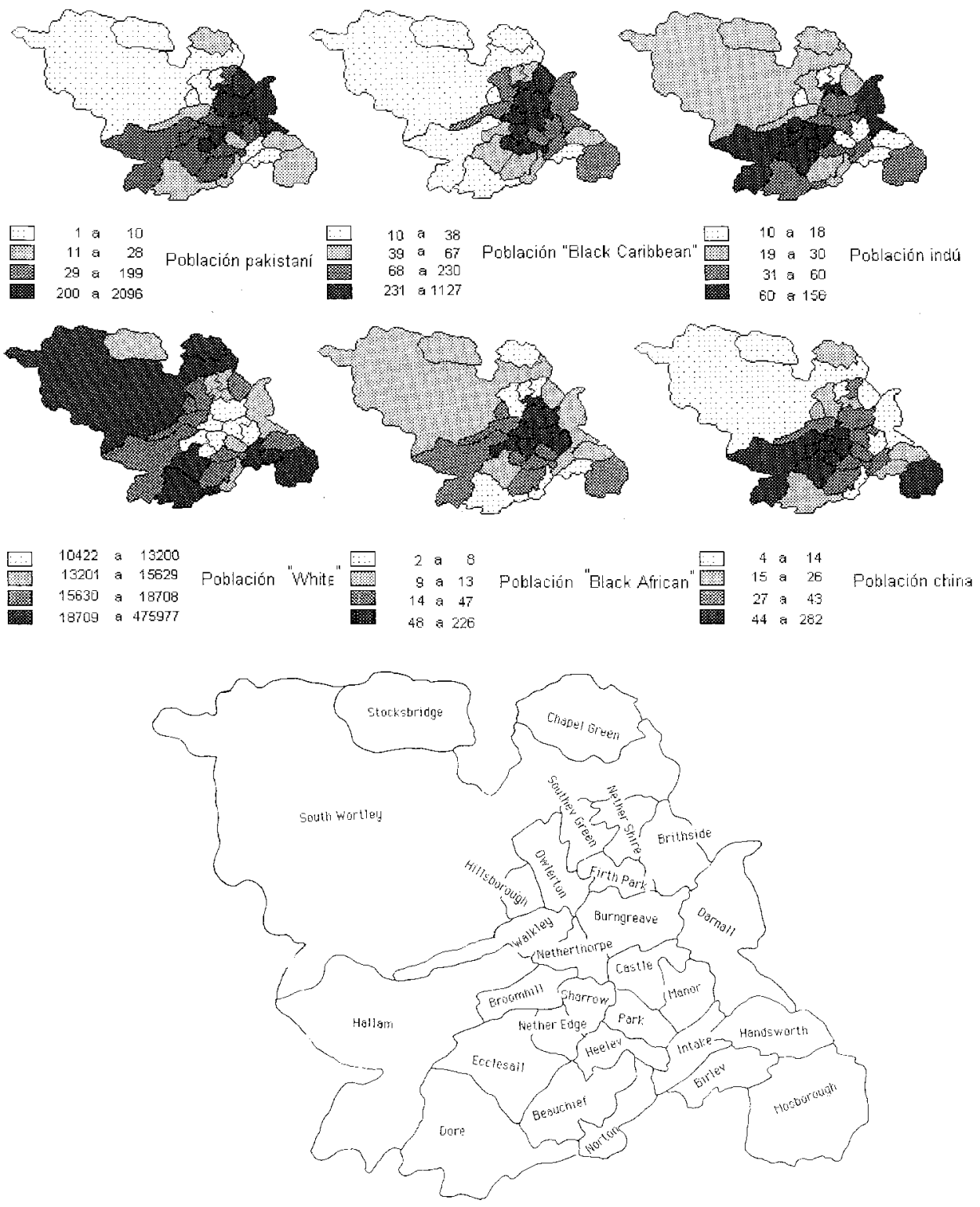

Figura 5.- Localización espacial: grupos étnicos en la ciudad de Sheffield. Fuente: Sheffield Census-1991. Elaboración propia. 
No se da segregación espacial propiamente dicha aunque sí cierta concentración que es mayor en los Asian Groups, Black African, Black Caribbean, Pakistani y Bangladeshi y mucho menor en la Comunidad White. El 70'17\% de la población de la Comunidad Pakistani vive en cuatro áreas principales-Darnall, Burngreave, Firth Park y Sharrow- y el 82'13\% de Bangladeshi en Darnall, Castle, Sharrow y Nether Edge, mientras que la población de las cuatro áreas principales de la Comunidad White: Mosborough, Chapel Green, South Wortley, Ecclessall, representa solo el 20'6\% de su total en la ciudad.

\section{ALGUNOS PROBLEMAS DE LAS MINORIAS ETNICAS}

El estudio de las Comunidades étnicas minoritarias de Sheffield nos aproxima no sólo al conocimiento de sus rasgos demográficos, socio-económicos, culturales y de localización en el espacio urbano, sino también a la problemática en la que a menudo se encuentran inmersas estas Comunidades. El racismo y la discriminación, el desempleo, la salud, la vivienda y la pérdida de las tradiciones culturales son algunos de los grandes problemas que, en ocasiones, padecen estas poblaciones.

Un trato oficial diferente $y$ algunas actitudes públicas denotan síntomas de racismo y discriminación. Este comportamiento no es algo nuevo en el R.U. pues ya se registraron ataques a ciudadanos de color en Liverpool, Londres y Glasgow después de la Primera Guerra Mundial hasta el punto de que el propio gobierno británico llegó a reconocer como problema, en 1981/82, los ataques raciales. Sheffield no es una excepción, tan solo de Abril a Octubre de 1991 se contabilizaron mas de 50 incidentes con violencia verbal o física.

Cuatro fenómenos principales, los abusos verbales, la discriminación en niños, la conducta abusiva y las amenazas gráficas $\mathrm{u}$ orales son algunas de las muestras racistas que representan alrededor de un $70 \%$ del total de incidentes. Más recientemente, de Enero a Marzo de 1994 hubo algo más de 100 denuncias por discriminación y ataques raciales efectuados en diferentes lugares: en la calle, en el trabajo, en casa, en las escuelas, etc lo que significa un aumento de denuncias respecto a las ocurridas en periodos anteriores sin contar las que no se llevan a cabo y se mantienen en secreto. Las dificultades para la búsqueda de los agresores son enormes y solamente se solucionan la mitad de los casos.

Es muy frecuente que tanto agresores como agredidos sean jóvenes; así por ejemplo, del $76 \%$ de los casos en que es conocido el agresor, durante el período AbrilOctubre de 1991, el 6\% es menor de14 años, el 37\% tienen de 14 a 16 años, un 16`5\% de 17 a 25 años y otro $16^{`} 5 \%$ más de 26 años. Tanto las agresiones verbales como los ataques y 
la discriminación racial es frecuente que sucedan en las escuelas entre alumnos, y en los lugares de trabajo donde se contrata preferentemente a trabajadores de la Comunidad White.

El desempleo afecta de modo desigual a los diversos grupos étnicos y a la población masculina y femenina. Es la población masculina de las comunidades BlackAfrican (40'55\%), Pakistani (39'15\%) y Bangladeshi (38'88\%) una de las más afectadas, y también la femenina de las citadas Comunidades Pakistani $(4178 \%)$ y Bangladeshi $\left(39^{\prime} 43 \%\right)^{3}$. Por el contrario, en el caso de los varones la Comunidad Chinese $\left(8^{\prime} 14 \%\right)$ es la menos afectada, y en el caso de las mujeres la Comunidad White (7'36\%). Es éste un importante problema para algunos grupos étnicos minoritarios como reconocen diferentes asociaciones de la ciudad, aunque la intensidad del mismo varíe entre unos y otros: bajos valores en la Comunidad Chinese y altos en la Pakistani y Bangladeshi.

- Salud y vivienda: Según las autoridades encargadas de la sanidad en la ciudad, los problemas de salud afectan de modo más evidente a las minorías étnicas que a la Comunidad White. En ello influye el desempleo, las dificultades con el lenguaje, las diversas formas de discriminación, los altos valores de pobreza frecuente; en definitiva, la suma de toda una serie de elementos que directa e indirectamente les afectan.

Cada Comunidad se ve afectada por problemas específicos: así por ejemplo, en el caso de los asiáticos, se da mayor incidencia de enfermedades como la diabetes y las del corazón entre varones y las depresiones entre las mujeres. La Comunidad con mayor incidencia de enfermedades de larga duración es la de los procedentes de Irlanda, mientras es menor en las Comunidades Chinese y Other Asian.

Las condiciones de vivienda de los grupos étnicos minoritarios, medidas a través de varios indicadores de confort: disponibilidad de ducha o bañera, calefacción, automóvil, etc... son deficientes. Los que cuentan con un menor número de servicios tales como ducha-bañera son la Comunidad Chinese (63\%), Other Asian (47\%) y Black African (45\%), en contraste con otros grupos con valores mucho menores como 1 a Comunidad White (1'7\%).

Carecen de calefacción, el 31\% y 29'9\% de las Comunidades Pakistani y Bangladeshi. Un elevado porcentaje de Black African (69'08\%), Black Caribbean (63'17\% y Black Other (56 65\%) no poseen automóvil, produciéndose un elevado número de personas por vivienda en la Pakistani y Bangladeshi. En cambio, las condiciones son

3 Los porcentajes no se refieren al total general de población sino al total de población action de cada grupo étnico. 
en general mejores entre la Comunidad White que es la más numerosa, como se ha mencionado con anterioridad.

Racismo y discriminación tienen a veces conexión con los problemas de la vivienda. El número de quejas registradas en el Departamento de la vivienda a lo largo del año 1993, fueron 93 tanto referidas a dificultades para encontrar una vivienda como por las deficientes condiciones de las mismas.

- Pérdida de tradiciones culturales: ¿Están siendo absorbidas las minorías étnicas por una cultura mayoritaria? Un anciano Pakistani fue muy expresivo a 1 respecto:"yo quiero volver a mi país pero mis hijos rehusan hacerlo, los chicos han comenzado a decirnos que cesemos de hablar nuestra lengua de Pakistán porque no comprenden". Ciertamente hay generaciones nacidas en el Reino Unido que no se preocupan del mantenimiento de la cultura tradicional que tiene sus raices en otros países, pero hay también jóvenes que buscan mantenerla y/o recuperarla. La antigüedad y origen de las corrientes migratorias y la actitud de los jóvenes pesa en el valor dado a la cultura tradicional.

\section{CONCLUSIONES}

La ciudad inglesa de Sheffield es un buen ejemplo de la heterogeneidad étnica que tiende a crecer cada vez más en las áreas urbanas europeas, debido a la importancia creciente de la movilidad internacional de la población. Las diferencias entre la Comunidad nativa, mayoritaria, denominada White y los grupos minoritarios que representan el $5^{\prime} 04 \%$ de la población total, se reflejan en sus características demográficas, sociales, económicas y culturales y en una localización espacial diferenciada en el plano de Sheffield.

La tendencia al envejecimiento es la característica demográfica más sobresaliente del grupo mayoritario, a diferencia de la mayoría de Comunidades mucho menos numerosas que tienen una considerable población joven. Se constatan diferencias sociales que tienden a acortarse a medida que aumentan las uniones interétnicas, a excepción de las Comunidades asiáticas que suelen ser más cerradas.

Fn el plano económico, la situación varia entre Comunidades y grupos de población, aunque globalmente el problema del desempleo afecta por igual a varones y mujeres en las Comunidades Black African, Pakistani y Bangladeshi. La población retirada procede sobre todo del grupo White y los estudiantes de grupos de origen asiático como la Comunidad China o Hindú. 
Culturalmente, la existencia de 47 lenguas es una buena muestra de la diversidad de la población inmigrada y de que los inmigrantes extranjeros a pesar del carácter oficial de la lengua inglesa, encuentran medios para mantener su lengua nativa.

La diversidad caracteriza también la distribución espacial de las minorías étnicas en Sheffield. Se encuentran áreas de cultura mixta y áreas dominadas por $\mathbf{u n}$ grupo étnico-cultural como en los barrios Bangladeshi o Jamaicano, que no constituyen por el momento espacios de segregación ni de exclusión.

Sin embargo, algunos problemas comunes afectan en diferente grado a todas las minorías étnicas: el racismo y la discriminación a los Black; el desempleo sobretodo a grupos de Pakistán y Bangladesh ; los problemas de salud y vivienda resultan más frecuentes también en estas dos últimas Comunidades y en las africanas y jamaicanas ; siendo la pérdida de las costumbres tradicionales un motivo de polémica interna general entre las diferentes generaciones que las componen.

En definitiva, las minorías étnicas forman parte del contingente inmigratorio en la población de Sheffield y aunque numéricamente no alcanzan cifras considerables constituyen un componente muy representativo de la nueva sociedad que se está configurando en las áreas urbanas europeas.

\section{BIBLIOGRAFIA}

BAIMBRIDGE M. et al. (1994): "The Maastricht Treaty: exacerbating racism in Europe?". Ethnic and Racial Studies. 17, 3, 420-436.

BERRINGTON, M. (1995): "Marriage and family formation among the White and ethnic minority population in Britain". Ethric and Racial Studies, 17, 3, 517-544.

C.P.S.C. (1993): 1991 CENSUS. Country report: South Yorkshire(Part.1/2). Ed.C.P.S.C. London.

CHAMPION, T. \& FIELDING, T. (Eds.) (1992): Migration processes patterns. Vol. I. Belhaven Press. London.

GARSON J.P. (1992): Migrations internationales: faits, chiffres, politiques. L'Observateur de L O.C.D.E. Organisation de Cooperation et de Developpement Economique. Paris.

HIGUERAS, A.(1981): "Reflexiones acerca de los movimientos de población". Geographicalia, .9. Institución Fernando El Católico-Instituto de Geografía Aplicada. C.S.I.C-Departamento de Geografía. Universidad de Zaragoza.

KING , R. (Ed.) (1993): Mass migrations in Europe. Ed. Belhave. 65-82. London. 
THUMERELLE P.J.(1992): Migrations internationales et changement geopolitique en Europe. Annales de Geographie , 565, 289-318. Ed.Armand Colin. Paris.

WHITE P. (1993): "The social geography of immigrants in European cities: the geography of arrival". En, R. King (Ed.)(1993): The new Geography of European Migration. Ed.Belhaven. London.

VV.AA (1994): Ethnic origin in Sheffield. Repport 9/Census 1991. Ed. Central Policy Unit. Sheffield 